\title{
Encabezamiento. DETERMINACION HISTOLOGICA DE CAMBIOS PRO- LIFERATIVOS ATIPICOS EN BIOPSIAS DE GLANDULA MAMARIA EN MUJERES USUARIAS DE IMPLANTES MAMARIOS PIP.
}

\section{DETERMINATION OF ATYPICAL PROLIFERATIVE CHANGES IN BIOPSIES OF THE BREAST TISSUE OF FEMALES WITH PIP MAM- MARY IMPLANTS}

\begin{tabular}{r} 
Marta Patricia Cabrera Cadavid $^{1}$ \\
\hline${ }^{1}$ Docente de Histología y Patología, Programa de Medicina, Facultad de Ciencias de la Salud, Universidad del Quindío. \\
Recibido: Junio 15 de 2015 \\
Aceptado: 16 de Agosto de 2015 \\
*Correspondencia del autor: Marta Patricia Cabrera Cadavid, Dirección: Calle 1 norte \# 12-36 Consultorio 529 Clínica Armenia, \\
E-mail: mapaca33@hotmail.com
\end{tabular}

\section{RESUMEN}

En Colombia en el año 2012 se generó pánico en las mujeres usuarias de prótesis PIP por la información sobre el riesgo de ruptura de estas prótesis ya que contenían silicona industrial y podían generar cáncer en las mujeres que las usaban.

Objetivo. Determinar la presencia de cambios proliferativos atípicos en biopsias de glándula mamaria en usuarias de prótesis de silicona.

Metodología. Se realizó un estudio descriptivo de corte transversal de los cambios histopatológicos encontrados en biopsias tru-cut bilateral de las glándulas mamarias previo consentimiento informado a 50 mujeres portadoras de prótesis mamarias, que acudieron para el cambio de dichas prótesis a una clínica de cirugía plástica en la ciudad de Armenia Quindío, durante el periodo marzo a diciembre del año 2012.

Se sistematizó la información de los datos en Excel y se realizó el análisis estadístico en programa de statgraphics centurión 1.15 .

Resultados. Se realizaron biopsias a 50 mujeres usuarias de prótesis de ambas glándulas mamarias, el tejido fue representativo en ambas mamas en 29 de ellas (58\%), en 3 usuarias (6\%) se obtuvo sólo tejido fibroadiposo en ambas mamas, en 9 (18\%) sólo se estudió el tejido de la mama derecha y en 12 (24\%) mujeres el de la mama izquierda pues las biopsias contralaterales mostraron tejido adiposo.

En ninguna biopsia se encontró cambios proliferativos atípicos, en 6 usuarias (12\%) se encontró cambio fibroquístico y en las restantes 41 usuarias $(82 \%)$, el tejido mamario fue normal.

Palabras Clave: Implantes de Mama, Enfermedad Fibroquística de la mama, hiperplasia, mama. 


\begin{abstract}
In 2012 in Colombia, there was panic among women with mammary prosthesis (PIP) because of a news report that explained that they contained industrial silicon, a product that could cause cancer in the women that used them.
\end{abstract}

Objective. To determine the presence of atypical proliferative changes in the biopsies of the mammary glands of women recipients of the prosthesis.

Methodology. A descriptive study that took a cross-section of the population affected was conducted on the histopathological changes found in bilateral, tru-cut biopsies of the mammary gland with the previous informed consent of fifty women patients that were recipients of the mammary prosthesis. All had requested that their prosthesis be changed at the Clinic of Plastic Surgery in the city of Armenia, Quindio, Colombia during the period from March to December 2012. Their information was entered into an Excel data base and a statistical analysis was done by Statgraphics Centurion 1.15

Results. Biopsies were conducted of both mammary glands of fifty women prosthesis users. The tissue was representative in both breasts in 29 of the women $(58 \%)$, in 3 users $(6 \%)$ fibroadipose tissue was found in both breasts, and in $9(18 \%)$ patients only tissue from the right breast was studied. In twelve users $(24 \%)$, only the tissue of the left breast was studied because the contralateral biopsie showed adipose tissue. There were not any atypical proliferative changes in any of the women. In 6 users (12\%), fibrocystic changes were found. In the remaining 41 users $(82 \%)$, the mammary tissue was normal.

Keywords: Breast implants, Fibrocystic Breast Disease, hyperplasia, Breast.

\section{INTRODUCCIÓN}

Los implantes mamarios se dirigen a mejorar o restablecer la morfología de la glándula mamaria, después de haber tenido previamente la privación de la mama como consecuencia de una mastectomia radical por cáncer o para aumentar el volumen en mujeres que lo precisan por anomalías del desarrollo o por estética. La primera mamoplastia de aumento reportada en la literatura médica data de 1895 , quien transfirió un lipoma de la cadera de una paciente a la mama, para corregir un defecto de volumen después de la resección de un tumor (fibroma) (1).

Durante los siguientes 50 años los cirujanos implantaron diferentes tejidos como cartílago, materiales como parafina, silicona líquida o cuerpos sólidos con una gran cantidad de complicaciones como infecciones o fístulas, en otras ocasiones se produjeron embolias a pulmones o al sistema nervioso central de los fluidos inyectados (1).

La silicona (polidimetilsiloxano) se sintetizó por primera vez en 1938, en 1947 se utilizó para evitar la maceración de las heridas quirúrgicas en contacto con el agua.
Fue ampliamente utilizada en la Segunda Guerra Mundial, para aplicación industrial y militar, con ella se fabricaron catéteres endovenosos, lentes intraoculares, prótesis articulares, jeringas, marcapasos y válvulas cardiacas protésicas $(1,2)$.

En 1950 se inició el empleo de materiales inyectables como aceites, algunos derivados del petróleo, estos trajeron como resultado no solo alteraciones del contorno, de la textura, color de la piel, úlceras cutáneas, calcificaciones, migración de líquidos o pérdida del tejido mamario (2).

En 1962 se implantó la primera prótesis mamaria utilizando un gel denso de silicona y cápsula gruesa de superficie lisa, el material fue bien tolerado con tasas de ruptura baja, pero alta de difusión del gel o de contractura capsular está última es una lámina de tejido conectivo formada alrededor de las prótesis que se encarga de adherirse a ellas cubriéndola en su totalidad (2).

En 1972 se modificó la cubierta adelgazándola pero se evidenció un nuevo problema, la ruptura o filtración 
del contenido de la prótesis permitiendo que la solución salina empleada o el gel de silicón se difundiera hacia los tejidos, perdiendo el volumen y alterándose la forma de la prótesis (2).

En 1980 se crearon las prótesis con cubierta de poliuretano que reducían la posibilidad de contractura capsular pero rápidamente entraron en desuso por que el poliuretano se podía fragmentar en toluendiamina el cual producía tumores en ratones de laboratorio más no en humanos, además este tipo de implantes se adherían al tejido mamario logrando que su retiro fuera muy difícil, teniéndose que retirar tejido mamario normal en gran cantidad.

Posteriormente hubo un periodo de receso ya que se planteó que el uso de estas prótesis inducían tumores $\mathrm{y}$ enfermedades autoinmunes $(1,2,3)$.

A final de la década de los noventa hasta el 2004 aparecen las prótesis de cuarta generación con gel cohesivo cubiertas con elastómero de silicón con superficies que disminuyen la posibilidad de contracción de la cápsula de tejido conectivo, además ésta cubierta no permite la filtración y el gel tiene alto grado de cohesividad lo que permite resultados más naturales (3).

La envoltura es una goma de silicona que puede ser simple o doble, lisa o texturizada o cubierta con espuma de poliuretano, el contenido puede ser realizado con gel de silicona o con solución salina normal(3).

Para la cuarta generación se introdujo la envoltura texturizada con el fin de disminuir la contractura capsular y se amplió la cohesividad del gel mejorando la forma y sensación de los implantes, a partir del años 2005 fueron realizados cambios significativos en las capas externas de las envolturas, actualmente los implantes de quinta generación tiene una envoltura que puede ser sencilla o doble, lisa o texturizada y se puede o no cubrir con poliuretano, la variación en la composición y características del elastómero de silicona en la envoltura se ha introducido para disminuir la hemorragia y aumentar la biodurabilidad del implante además la superficie texturizada promueve el crecimiento de las células del intersticio y desorienta las fibras de colágeno, debilitando las fuerzas contractuales, generando una inflamación crónica con el objetivo de disminuir la contractura capsular ( 4 ).

Los implantes mamarios de silicona PIP fueron fabricados por la empresa Poly Implant Prothese ( PIP), creada en 1991 en el sur de Francia recibiendo los registros sanitarios de éste país y en 1997 fueron aprobados por la European Unión Medical Devices Directive, en el Reino Unido se aprobaron en el año 2000 , tras reportes recientes de ruptura de estas prótesis desde el año 2007 las agencia Francesa y del Reino Unido iniciaron investigaciones encontrando irregularidades en la calidad y en los materiales de fabricación de los implantes razón por la que en marzo del 2010 se ordenó la suspensión de la producción, comercialización, exportación y uso de estas prótesis (4).

En Colombia el Invima dio registro de autorización en Junio de 1999 y lo renovó el 5 de Marzo del 2009 a pesar que ya aparecían los primeros reportes de ruptura, en el examen de microscopia óptica al elastómero de silicona de la envoltura se le encontró irregularidades como una distribución no homogénea y al parecer la envoltura era de fabricación rústica y no de doble barrera, lo que además predispone a la difusión crónica del gel (4).

PIP recibió registro para la fabricación con las características de los implantes de cuarta generación utilizando como gel de relleno el gel nusil, con las investigaciones se estableció que el gel de relleno fue un gel de silicona industrial utilizado para la fabricación de colchones, almohadas, componentes electrónicos o de la industria del caucho, también se han reportado el aislamiento de algunos derivados del petróleo como baisilona, silopreno y rodorsil, el gel de relleno de estas prótesis tienen disminución en las propiedades biscoelásticas al ser menos cohesivo por lo que tiene mayor tendencia a dispersarse o migrar localmente. (4).

La silicona industrial de estas prótesis tienen mayor potencial irritante lo que genera reacciones locales, los estudios histológicos de la cápsula y del tejido mamario que ha estado en contacto con estas prótesis han mostrado infiltración de células inflamatorias crónicas con células gigantes, granulomas, zona de necrosis y fibroesclerosis difusa pero sin evidencia de malignidad (4).

Epidemiologicamente se conoce que los principales factores de riesgo para desarrollar cáncer de mama son los hormonales y genéticos, los carcinomas esporádicos son probablemente relacionados con la exposición a hormonas, el sólo hecho de ser mujer es un 
factor de riesgo para presentarlo, al igual que la edad, a mayor edad aumenta la incidencia, las mujeres que presentan la menarquía antes de los 11 años tiene un aumento en el riesgo del $20 \%$ en comparación con las que llegan a la menarquía después de los 14 años. La menopausia tardía también aumenta el riego, las mujeres que experimentan un embarazo a término antes de los 20 años tienen la mitad de riesgo que las mujeres nulíparas o aquellas que tienen hijos por primera vez después de los 35 años (5).

El riesgo de cáncer de mama aumenta con el número de familiares en primer grado afectados ( madre, hermana o hija), sobre todo si el cáncer ocurrió a una edad temprana. Sólo el 13\% de las mujeres con cáncer de mama tienen un familiar en primer grado afectado y sólo $1 \%$ tienen dos o más. Es probable que la mayor parte del riesgo familiar se deba a la interacción de genes de susceptibilidad de riesgo bajo y de factores no genéticos. (5).

La exposición a estrógenos ya sea por tratamiento hormonal de reemplazo o por producción endógena de éstos a partir de los depósitos de grasa como en la mujeres obesas post-menopáusicas aumentan el riesgo, la exposición a los estrógenos impulsa ciclos de proliferación que determinan un riesgo de daño al ADN en las células, si existen células atípicas o malignas, las hormonas pueden estimular su crecimiento, además los metabolitos de los estrógenos pueden causar mutaciones o generar radicales libres dañinos para el ADN en las células $(5,6,7)$.

Los factores hereditarios se asocian a la mutación de un gen con alta penetrancia, las mutaciones de los genes BRCA 1 y BRCA2 son responsables de la mayoría de los canceres atribuibles a mutaciones únicas, pero estos genes de alto riesgo son responsables de sólo alrededor de una cuarta parte de los cánceres de mama familiares $(5,8)$.

Con relación a la carcinogenia y progresión tumoral en mama, la más temprana de estas alteraciones consiste en cambios proliferativos que pueden preceder de la pérdida de señales inhibitorias de crecimiento, aumento de las señas favorecedoras del crecimiento o disminución de la apoptosis, la mayoría de las lesiones tempranas como hiperplasia epitelial ductal o lobulillar atípica muestran una expresión aumentada de receptores de hormonas y una regulación anormal de proliferación. $(9,10)$.
Las mujeres con cambios hiperplasia ductal o lobulillar atípica presentan un riesgo de carcinoma invasivo cuatro o cinco veces mayor que la población general (11).

El colegio americano de patólogos recomendó agrupar las mujeres con cambio fibroquístico en tres categorías para la probabilidad de riesgo de cáncer.

- Ausencia de hiperplasia o hiperplasia epitelial leve: no se observa riesgo de carcinoma invasivo.

- Hiperplasia epitelial moderada o florida: 1,5 a 2 veces de presentar riesgo.

- Hiperplasia epitelial ductal, o lobulillar atípica: 5 veces de presentar el riesgo $(8,11,12,13)$.

El uso de material no adecuado y no certificados para la fabricación de las prótesis a generado un alerta mundial, en el país a comienzo del año 2012 las usuarias de prótesis marca PIP fueron alertadas sobre el riesgo de ruptura de estas lo que podía causar mayor predisposición a complicaciones tales como el cáncer creando angustia y temor, el cual fue incrementado por los medios de comunicación que han tenido un papel transcendental divulgando diferentes teorías y complicaciones, muchas veces sin fundamentos científicos.

El presente estudio determina mediante biopsia de tejido de usuarias de próstesis, si existe o no cambios proliferativos atípicos en el parénquima mamario de estas mujeres.

\section{Metodología}

Previo consentimiento informado se realizaron biopsias tru-cut de los cuadrantes externos de ambas glándulas mamarias a 50 mujeres portadoras de prótesis durante el periodo marzo a diciembre del año 2012 que acudieron a una Clínica de cirugía plástica de la ciudad de Armenia Quindío, para el cambio de dichas prótesis, en este consentimiento se explica que la biopsia era realizada con el fin de obtener un estudio microscópico del tejido mamario para detectar precozmente alteraciones histopatológicas.

Se realizó un estudio descriptivo de corte transversal, se sistematizó la información de los datos en Excel y se realizó el análisis estadístico en programa de Statgraphics Centurión1.15.

Las biopsias se tomaron durante el tiempo quirúrgico, se procesaron e interpretaron en laboratorio de patología particular por médica patóloga, quien proporcionó 
un diagnóstico en un término de 10 días hábiles después de realizadas, entregándose el resultado a cada usuaria durante los controles posteriores realizados por el cirujano plástico.

En el proceso de consentimiento se informó que la biopsia y el estudio microscópico no tenían costo adicional, las complicaciones y en caso de encontrarse alteraciones histopatológicas en los reportes de las biopsias, las usuarias serían notificadas oportunamente y se remitirían a las EPS correspondientes para seguir el tratamiento pertinente.

Se llevo a cabo encuestas para determinar variables como la edad, tiempo de uso de las prótesis, historia de ruptura y tiempo después de implantadas, número de veces que se ha presentado la ruptura de las prótesis, realización de estudios radiológicos para saber el estado del tejido mamario y de las prótesis, periodicidad de los mismos, historia familiar de cáncer mamario y el parentesco, el conocimiento y realización del auto examen de la glándula mamaria.

El estudio histopatológico determinó si el tejido obtenido era adecuado para estudio histológico, enten- diéndose por adecuado el que la muestra obtenida presentara conductos y acinos mamarios, la muestra no es adecuada cuando sólo se obtuvo tejido fibroadiposo.

Se evaluó la presencia de alteración fibroquística (alteración no proliferativa), cambio proliferativos sin atípia y enfermedad proliferativa con atípia.

\section{Resultados}

A todas las usuarias del estudio se les realizó biopsia tru-cut de ambas glándulas mamarias,el tejido mamario fue representativo para estudio en $29(58 \%)$ usuarias en ambas mamás, en 9 mujeres (18\%) sólo fue posible evaluar la mama derecha y en $12(24 \%)$ la glándula mamaria izquierda ya que la biopsia contralateral sólo presentaba tejido adiposo o fibroadiposo sin acinos o conductos mamarios.

En 6 usuarias (12\%) se encontró cambio fibroquístico, $3(6 \%)$ presentaron en sus biopsias bilaterales tejido adiposo y en las $41(82 \%)$ restantes el tejido mamario fue histológicamente normal, sin evidencia de cambio proliferativos o atípia, de estas 41 usuarias, 12 (24\%) tenían solo tejido adiposo en la biopsia de mama derecha y en 9 usuarias (18\%) en la mama izquierda.

\begin{tabular}{|c|c|c|c|c|}
\hline \multicolumn{5}{|c|}{ TABLA 1: VARIABLES CUANTITATIVAS DEL ESTUDIO } \\
\hline VARIABLE & VALOR & FRECUENCIA & $\%$ & $\begin{array}{l}\text { FRECUENCIA } \\
\text { RELATIVA }\end{array}$ \\
\hline \multirow{4}{*}{$\begin{array}{l}\text { Presencia de tejido adi- } \\
\text { poso en la biopsia }\end{array}$} & Ambas & 3 & $6 \%$ & 0,06 \\
\hline & Derecha & 12 & $24 \%$ & 0,24 \\
\hline & Izquierda & 9 & $18 \%$ & 0,18 \\
\hline & No & 26 & $52 \%$ & 0,52 \\
\hline \multirow{3}{*}{ Biopsia representativa } & Ambas mamas & 29 & $58 \%$ & 0,58 \\
\hline & Derecha & 9 & $18 \%$ & 0,18 \\
\hline & Izquierda & 12 & $24 \%$ & 0,24 \\
\hline \multirow{4}{*}{$\begin{array}{l}\text { Cambio fibroquístico en } \\
\text { la biopsia }\end{array}$} & No & 41 & $82 \%$ & 0,82 \\
\hline & Sí & 6 & $12 \%$ & 1 \\
\hline & No representativa & 3 & $6 \%$ & 0,06 \\
\hline & No & 47 & $94 \%$ & 0,94 \\
\hline $\begin{array}{l}\text { Cambio proliferativo } \\
\text { atípico en la biopsia }\end{array}$ & No representativa & 3 & $6 \%$ & 0,06 \\
\hline
\end{tabular}

En la tabla 1 se puede observar los resultados de cada una de las variables cuantitativas presentadas en frecuencia, porcentaje y frecuencia relativa con respecto al estudio histopatológico de las biopsias de mama.

Los antecedentes familiares de cáncer mamario fueron descritos en 8 usuarias (16\%).

En cuanto a el parentesco: 6 (12\%) usuarias manifestaron como antecedentes familiares una tía, 1 usuaria
(2\%) la abuela y una usuaria (2\%) manifestó como antecedente una prima.

Sobre el conocimiento de como realizarse el autoexamen, 34 usuarias (68\%) manifestaron que sí lo sabían 
realizar y $16(32 \%)$ no.

En cuanto a la frecuencia de periodicidad de los estudios radiológicos: 24 (48\%) usuarias se realizaron dos exámenes radiológicos y 16 (32\%) pacientes un examen radiológico.

El tipo de examen practicado fue ecografía realizadas por $33(66 \%)$ usuarias, 7 (14\%) mamografía, 5 (10\%) mamografía y resonancia magnética, una usuaria (2\%) sólo resonancia y 4 (8\%) pacientes no refirieron ningún examen.

Los resultados de los estudios radiográficos en 30 $(60 \%)$ mujeres fueron reportados normales, en 9 (18\%) se encontró prótesis rota, en 5 usuarias $(10 \%)$ presencia de quistes, $5(10 \%)$ usuarias no refiere el resultado, y en una usuaria (2\%) se encontraron microcalcificaciones.

\begin{tabular}{|c|c|c|c|c|c|}
\hline \multicolumn{6}{|c|}{ TABLA 2: OTRAS VARIABLES CUANTITATIVAS DEL ESTUDIO } \\
\hline VARIABLE & VALOR & FRECUENCIA & $\%$ & FRECUENCIA & RELATIVA \\
\hline \multirow{3}{*}{$\begin{array}{l}\text { Antecedentes fami- } \\
\text { liares de cáncer de } \\
\text { mama }\end{array}$} & No & 42 & $84 \%$ & 0,84 & \\
\hline & Sí & 8 & $16 \%$ & 0,16 & \\
\hline & Ninguno & 42 & $84 \%$ & 0,84 & \\
\hline \multirow{3}{*}{ Parentesco } & Tía & 5 & $10 \%$ & 0,1 & \\
\hline & Abuela & 1 & $2 \%$ & 0,02 & \\
\hline & Prima & 2 & $4 \%$ & 0,04 & \\
\hline \multirow{2}{*}{$\begin{array}{l}\text { Frecuencia de ruptura } \\
\text { de la prótesis }\end{array}$} & No & 32 & $64 \%$ & 0,64 & \\
\hline & $\mathrm{Si}$ & 18 & $36 \%$ & 0,36 & \\
\hline \multirow{3}{*}{$\begin{array}{l}\text { Realización de exá- } \\
\text { menes radiológicos }\end{array}$} & No & 4 & $8 \%$ & 0,08 & \\
\hline & Sí & 46 & $92 \%$ & 0,92 & \\
\hline & Ecografía & 33 & $66 \%$ & 0,66 & \\
\hline \multirow{4}{*}{$\begin{array}{l}\text { Tipo de examen } \\
\text { radiológico }\end{array}$} & Mamografía & 5 & $10 \%$ & 0,1 & \\
\hline & Mamografía y Ecografía & 7 & $14 \%$ & 0,14 & \\
\hline & Ninguno & 4 & $8 \%$ & 0,8 & \\
\hline & Resonancia & 1 & $2 \%$ & 0,2 & \\
\hline \multirow{7}{*}{$\begin{array}{l}\text { Resultado de estudios } \\
\text { radiológicos }\end{array}$} & Microcalcificaciones & 1 & $2 \%$ & 0,02 & \\
\hline & No se realizaron & 4 & $8 \%$ & 0,08 & \\
\hline & Normal & 30 & $60 \%$ & 0,6 & \\
\hline & No reporta & 1 & $2 \%$ & 0,02 & \\
\hline & Prótesis rota & 9 & $18 \%$ & 0,18 & \\
\hline & Quistes & 5 & $10 \%$ & 0,1 & \\
\hline & No & 16 & $32 \%$ & 0,32 & \\
\hline Autoexamen & Sí & 34 & $68 \%$ & 0,68 & \\
\hline
\end{tabular}

En la tabla 2. Observamos el resultado de otras variables cuantitativas analizadas en el estudio.

El promedio de edad de las usuarias de las prótesis fue de 37 años, con una desviación estándar de 9.03, un mínimo de 22 años y un máximo de 61 años.

El tiempo de uso de las prótesis tuvo un promedio de 5 años, con un mínimo de 3 años y un máximo de 16 años, 26 mujeres (52\%) usuarias las han portado por 3años y $16(32 \%)$ usuarias por 6 años, sólo una de

ellas (2\%) la ha usado por más de 16 años.

Se presentó ruptura de una prótesis en 18 usuarias (36\%) con un promedio de 5años de uso, un mínimo de 1 año y un máximo de 13 años, 32 mujeres (64\%) nunca tuvieron ruptura de las prótesis.

$39(78 \%)$ usuarias se realizaron entre 1 a 2 estudios radiológicos para saber el estado de las prótesis.

En cuanto a la frecuencia de periodicidad de los estu- 
Determinación histológica de cambios proliferativos atípicos en biopsias de glándula mamaria. Cabrera.

dios radiológicos: 24 ( 48\%)usuarias se realizaron 2 exámenes radiológicos y 16 (32\%) pacientes un examen.

El número de veces de la ruptura de las prótesis cuan- do fue manisfestada fue de sólo una vez (2\%).

La marca de las prótesis reportadas en todas las usuarias que se estudiaron fue PIP $(100 \%)$.

\begin{tabular}{lccc}
\hline \multicolumn{4}{c}{ TABLA 3: VARIABLES CUALITATIVAS IDENTIFICADAS EN EL ESTUDIO } \\
\hline \multicolumn{1}{c}{ VARIABLE } & PROMEDIO & DESVIACIÓN ESTÁNDAR & IC 95\% \\
Edad (en años) & 37,68 & 9,03 & $35,02-40,33$ \\
Tiempo de uso(en años) & 5,94 & 2,78 & $5,14-6,73$ \\
Tiempo ruptura (en años) & 5,26 & 2,57 & $3,83-6,69$ \\
Periodicidad de exámenes radiológicos & 1,75 & 0,72 & $1,52-1,99$ \\
Número de veces de ruptura de la prótesis & 0,36 & 0,48 & $0,22-0,49$ \\
\hline
\end{tabular}

En la tabla 3, se reportan los resultados de las variables cualitativas del estudio presentadas en promedio, desviación estándar e intervalos de confianza 95\%.

\section{Discusión}

En Colombia el cáncer de mama es la tercera causa principal de muerte entre las mujeres después del cáncer de cuello uterino y el cáncer gástrico $(5,6)$

Con el incremento del uso de prótesis mamarías especialmente con fines estéticos y cada día a edad más temprana, se han realizado numerosos estudios e investigaciones si éstas son causantes de cáncer.

Los resultados de las biopsias realizadas solo demostraron cambio fibroquístico en 6 mujeres ( $12 \%$ ), no encontramos cambios epiteliales proliferativos atípicos, esto se relaciona con los múltiples estudios reportados en la literatura como los Deape, Berkel, Bryant, Silverstein y colaboradores etc, estudios retrospectivos donde determinan que la incidencia de cáncer mamario no se incrementa con el uso de implantes mamarios y el riesgo no es significativamente más alto o más bajo que el de la población general. $(14,15,16,17,18)$.

Ya es bien conocido que los principales factores de riesgo para el desarrollo de éste cáncer son los factores hormonales y genéticos. (5).

Con relación al estudio realizado la edad promedio de las usuarias de prótesis fue de 37 años con un mínimo de 22 y un máximo 61 años, en su mayoría usuarias jóvenes que no están en el rango de edad para presentar carcinoma de mama, el cual como lo reporta la literatura tiene mayor probabilidad de presentarse a mayor edad hasta un alcanzar un pico a los 75 a 80 años, especialmente en los carcinomas esporádicos que son los relacionados con la exposición a hormonas.(5).

Otros de los factores de riesgo implicado son los hereditarios, la herencia de uno o varios genes de susceptibilidad es la causa principal en aproximadamente un $12 \%$ de los cánceres mamarios la probabilidad aumenta cuando existen múltiples familiares de primer grado afectadas, tanto en el lado de la familia del padre o de la madre, cuando las mujeres se afectan antes de la menopausia, tienen múltiples cánceres o cuando se tienen miembros de la familia con otros cánceres específicos (6 y 7 ).

En el estudio sólo 8 mujeres manifestaron antecedentes familiares de cáncer de mama pero sus biopsias tampoco se encontraron alteraciones histopatológicas. Se observó una mujer que ha usado prótesis por más de 16 años y aunque ha presentado ruptura de las prótesis la biopsia fue negativa.

Bajo ciertas circunstancias, los implantes mamarios pueden dificultar la detección de cáncer mamario por mamografía $(19,20)$ disminuyendo su sensibilidad pero estudios más especializados como la resonancia magnética o el ultrasonido resultan una excelente alternativa, en el trabajo la mayoría de las usuarias se habían realizado exámenes radiológicos en un mayor porcentaje ecografía, seguida por la mamografía estos exámenes además la presencia de prótesis rota en 9 mujeres permitieron detectar otras alteraciones como quistes y microcalcificaciones.

La utilización de prótesis de aumento en mujeres cada día más jóvenes nos hace concientizar de que el auto examen es un método sencillo de ayuda diagnóstica para detección de cáncer y sin costo que debe rea- 
lizarse periódicamente en búsqueda de alteraciones, llama la atención que las encuestas realizadas el 32\% de ellas manifestaron no tener conocimiento de cómo realizarlo.

Conclusión. En el presente estudio no se encontró relación entre el uso de prótesis mamaria PIP y la presencia de cambios proliferativos atípicos en las biopsias de tejido mamario.

\section{Recomendaciones.}

Se recomienda para futuras investigaciones realizar biopsias de control a largo plazo, a usuarias que portaron prótesis PIP, especialmente si presentaron ruptura y a una edad más avanzada para poder determinar si hay diferencias con los hallazgos histopatológicos encontrados actualmente.

Se debe instar a los cirujanos plásticos suministrar una amplia información a la mujeres que van a ser portadoras de prótesis acerca de las medidas de prevención que deben tomar, dar a conocer la importancia del autoexamen y antes de realizar implantes mamarios conocer la historia familiar de cáncer especialmente de glándula mamaria, uso de terapia estrogénica,si ha tenido biopsias previas de mama con hiperplasia epitelial significativa para ser prudentes o reconsiderar el uso de implantes.

Conflicto de interés y financiación. La autora manifiesta no presentar ningún conflicto de interés con lo presentado en éste artículo. Así mismo, se informa que no contó con recursos específicos.

Agradecimientos. De manera especial, por su aporte y apoyo en el desarrollo del estudio a los profesionales de la salud,Dr Jorge Mario Gómez, Cirujano Plástico y a la Dra Olga Alicia Nieto, PhD en Ciencias Basicas.

\section{BIBLIOGRAFÍA}

1. Perea,J.E.. Protesis mamarias. susMedicos. 2001-2006;(3). Accedido julio 23,2014.Disponible en: www.susMedicos.com/art-historia-prótesis.htm.

2. kossovsky,N.,Freiman,C.J.Physiochemical and inmmunological basis of silicone pathophisiology.J.Biomater S Cl polimer EDN 1995; 7:101-113.

3. Cronin,T.D., Gerow F. Augmentation mammaplasty: a new natural feel prohtesis.Excerpta Medical International Congres Series. 1964; 66; 41-49.

4. AmayaF.,Mendoza M.B. El problema PIP, origen y consecuencias.Sociedad Colombiana de Cirugía Plástica Estética y Reconstructiva. 11 Diciembre 2014. Disponible en WWW.ciplastica.com/sccp11diciembre2014.

5. Kumar V.,Abbas.A. K.,Fausto N., Aster J. Patología estructural y funcional. 8a ed. España: ELSEVIER; 2010.

6. Piñeros P M.,Pardo R.C,,Gamboa O., Hernández S.G. Atlas de mortalidad por cáncer en Colombia. República de Colombia. Ministerio de Protección Social. I.N.C.,E.S.E. Bogotá. 2010 pag. 43. Accedido agosto4 2014.Disponible en: www.cancer.gov.co.

7. Liga Contra el Cáncer Seccional Bogota. Cáncer de seno.Liga contra el cáncer. 2006; (8). Accedido julio25,2014.Disponible en: www.ligacontraelcancer.com.co.seccional bogotá. cáncer de seno.

8. Rosai J. and Ackerman's. Surgical Pathology. 10a ed. New York: ELSEVIER; 2011.

9. Allred D. C., Wu Y.,Mao S.,Nagtegaal I.D.,Sangjun L.,Perou M.C.,et al. Ductal carcinoma in situ and the emergence of diversity during breast cancer evolution. Clin Cáncer Res. 2008; 14: 370-378.

10. Iqbal M.,Davies M.P.,Shoker B.S.,Jarvis C.,Sibson D.R.,Sloane J. P. Subgroups of non-atypical hyper- 
plasia of breast defined by proliferatión of estrogen receptor positive cell. J Pathol. 2001; 193: 333-338.

11. Fitzgibbons P. L. ,Henson D. E.,Hutter R. V. Benign breast changes and the risk for subsequent breast cáncer: an up date of the 1985 consensus statement.Cancer committe of the College of American Pathologist. Arch Pathol Lab Med. 1998; 122:1053-1055.

12. Schnitt S. J. Benign breast disease and breast cancer risk morphology and beyond.Am J Surg Pathol.2003; 27: 836-841.

13. Hartmann L C et al. Benign breast disease and the risk of breast cancer.N. Engl. J. Med. 2005; 353:229237.

14. Berkel H., Birdsell D. C., Jenkins H. Breast Augmentation.A risk factor for breast cancer?. N. Engl J. Med 1992; 326: 1649-1653.

15. Bryant H., Brasher P. Breast implants and breast cancer.Reanalysis of a linkage study. N Engl J Med. 1995; 332: 1535-1539.

16. Deapen D.M., Brody G.S. Augmentation mamoplasty and breast cancer: a 5 year update of the los angeles study.Plast.Reconst.Surg. 1992; 89:660-65.

17. Deapen D. M.,Pike M. C.,Casagrande J. T.,Brody G. S. The relationship between breast cancer and augmentation mammaplasty: an epidemiologig study. Plast Reconstr Surg 1986; 77:361-8.

18. Silverstein M.J., Handel N., Gamagani P., Waisman J.R., Gierson E. D. Breast cancer in women after augmentation mammoplasty. Arch Surg 1988; 123:681-5

19. Grace G. T., Roberts C.,Cohen I. K. The role of mammography in detecting breast cancer in augmented breast.Ann Plast Surg 1990; 25: 119-123.

20. Clark C.P., Peters G. N., O'Brienk M. Cancer in the augmented breast diagnosis and prognosis.Cancer $1993 ; 722170-2174$. 